\title{
Dysregulated bile acid receptor-mediated signaling and IL-17A induction are implicated in diet-associated hepatic health and cognitive function
}

Prasant Kumar Jena ${ }^{1,2}$, Lili Sheng ${ }^{1,3}$, Michelle Nguyen ${ }^{1}$, Jacopo Di Lucente', Ying Hu', Yongchun $\mathrm{Li}^{1,4,5}$, Izumi Maezawa', Lee-Way Jin ${ }^{1}$ and Yu-Jui Yvonne Wan $^{1 *}$ (D)

\begin{abstract}
Background: Chronic consumption of high sugar and high fat diet associated with liver inflammation and cognitive decline. This paper tests a hypothesis that the development and resolution of diet-induced nonalcoholic fatty liver disease (NAFLD) has an impact on neuroplasticity and cognition.

Methods: C57BL/6 wild-type mice were fed with either a healthy control diet (CD) or a fructose, palmitate, and cholesterol (FPC)-enriched diet since weaning. When mice were 3-months old, FPC diet-fed mice were randomly assigned to receive either FPC-enriched diet with or without $6 \%$ inulin supplementation. At 8 months of age, all three groups of mice were euthanized followed by analysis of inflammatory signaling in the liver and brain, gut microbiota, and cecal metabolites.

Results: Our data showed that FPC diet intake induced hepatic steatosis and inflammation in the liver and brain along with elevated RORY and IL-17A signaling. Accompanied by microglia activation and reduced hippocampal long-term potentiation, FPC diet intake also reduced postsynaptic density-95 and brain derived neurotrophic factor, whereas inulin supplementation prevented diet-reduced neuroplasticity and the development of NAFLD. In the gut, FPC diet increased Coriobacteriaceae and Enysipelotrichaceae, which are implicated in cholesterol metabolism, and the genus Allobaculum, and inulin supplementation reduced them. Furthermore, FPC diet reduced FXR and TGR5 signaling, and inulin supplementation reversed these changes. Untargeted cecal metabolomics profiling uncovered 273 metabolites, and 104 had significant changes due to FPC diet intake or inulin supplementation. Among the top 10 most affected metabolites, FPC-fed mice had marked increase of zymosterol, a cholesterol biosynthesis metabolite, and reduced 2,8-dihydroxyquinoline, which has known benefits in reducing glucose intolerance; these changes were reversible by inulin supplementation. Additionally, the abundance of Barnesiella, Coprobacter, Clostridium XIVa, and Butyrivibrio were negatively correlated with FPC diet intake and the concentration of cecal zymosterol but positively associated with inulin supplementation, suggesting their benefits.

(Continued on next page)
\end{abstract}

\footnotetext{
* Correspondence: yjywan@ucdavis.edu

'Department of Medical Pathology and Laboratory Medicine, University of California, Davis Health, Room 3400B, Research Building III, 4645 2nd Ave, Sacramento, CA 95817, USA

Full list of author information is available at the end of the article
}

(c) The Author(s). 2020 Open Access This article is licensed under a Creative Commons Attribution 4.0 International License, which permits use, sharing, adaptation, distribution and reproduction in any medium or format, as long as you give appropriate credit to the original author(s) and the source, provide a link to the Creative Commons licence, and indicate if changes were made. The images or other third party material in this article are included in the article's Creative Commons licence, unless indicated otherwise in a credit line to the material. If material is not included in the article's Creative Commons licence and your intended use is not permitted by statutory regulation or exceeds the permitted use, you will need to obtain permission directly from the copyright holder. To view a copy of this licence, visit http://creativecommons.org/licenses/by/4.0/ The Creative Commons Public Domain Dedication waiver (http://creativecommons.org/publicdomain/zero/1.0/) applies to the data made available in this article, unless otherwise stated in a credit line to the data. 
(Continued from previous page)

Conclusion: Taken together, the presented data suggest that diet alters the gut microbiota and their metabolites, including bile acids. This will subsequently affect IL-17A signaling, resulting in systemic impacts on both hepatic metabolism and cognitive function.

Keywords: Gut-liver axis, Gut-brain axis, Inflammation, Neuroplasticity, Metabolic syndrome, Gut microbiota, Bile acid receptor, Dementia, Cognition

\section{Introduction}

Non-alcoholic fatty liver disease (NAFLD) is associated with reduced cognitive performance in adults through the impairment of psychomotor speed and learning ability, suggesting a link between hepatic metabolism and brain function [1]. Additionally, there is an increased prevalence of metabolic syndromes in patients with NAFL $\mathrm{D}$, which is considered a risk of cognitive decline [2]. Moreover, early-life exposure to a Western diet (WD) impairs hippocampal-dependent memory and neuroplasticity [3]. This paper tests a hypothesis that the development and resolution of diet-induced NAFLD has an impact on brain neuroplasticity. We also studied whether highly fermentable fiber inulin can prevent WD-induced liver disease and WD-impaired neuroplasticity simultaneously.

Emerging evidence reveals the significance of diet via the gut microbiota in regulating liver health $[4,5]$. The gut microbiota also affects neuronal functions by modifying the production of vitamins, neurotransmitters, and neuroactive microbial metabolites such as short-chain fatty acids (SCFA) [6]. Additionally, the gut microbiome and brain communicate in a bidirectional manner, modulating the activation of microglial cells and affecting myelination as well as neurogenesis in the brain $[7,8]$. Thus, we aim to establish the gut microbiota and their associated metabolites implicated in the development of NAFL $\mathrm{D}$ and cognitive decline as well as their resolutions.

Gut dysbiosis alters the composition of the bile acid (BA) pool, resulting in compromised BA receptor farnesoid $x$ receptor (FXR) and Takeda G protein-coupled receptor 5 (TGR5)-regulated signaling [9]. Both FXR and TGR5 are expressed in the liver as well as the gut, and they are detectable in the brain where they regulate metabolism and immunity [10, 11]. Activation of TGR5 with its agonist INT-777 ameliorates synaptic dysfunction against $A \beta_{1-42}$-induced neurotoxicity [12]. BAs also regulate the gut microbiota directly via their antibacterial activity [9]. Additionally, the secondary BAs are bacterial metabolites, which are implicated in liver carcinogenesis and cognitive decline [13, 14]. Therefore, BAs and their regulated signaling explains how diet via the gut microbes affects health at the systemic level.

Inulin is a nondigestible fiber present in a wide variety of fruits and vegetables. It is commonly used as a dietary supplement for weight loss and lowering serum lipids
[15]. Through gut microbial fermentation, inulin generates SCFAs that have health benefits [16]. It has been shown that inulin improves mood as well as memory and digestion in humans [17]. Inulin also ameliorates castration-induced cognitive decline [18]. Unexpectedly, although long-term (6 months) supplementation of inulin (7.5\%) improves metabolic syndrome in toll-like receptor 5 (TLR5)-knockout mice, many of these mice develop icteric hepatocellular carcinoma (HCC) [19]. These surprising findings suggest that long-term consumption of inulin is a potential risk for developing liver disease; therefore, there is a need to develop precision dietary supplementation [20]. Together, this study aims to further understand the impact of inulin through altering gut microbes and metabolites, which thereby affects liver and brain health.

In contrast to our previously used WD (TD.140414) [4, 21], which has a moderate amount of fat and high sucrose, we examined the long-term (7 months) effects of another diet named "fast food diet" or fructose, palmitate, and cholesterol (FPC) diet on liver and brain health in current study. FPC diet has been used to induce severe liver fibrosis and non-alcoholic steatohepatitis (NASH) [22]; however, its effects on the brain have never been studied. Our data show that different Western diets that contain enriched sugar and fat consistently compromised neuroplasticity and induced NAFLD. However, inulin supplementation concomitantly prohibited the negative effects of those WD in the brain and liver. Furthermore, gut microbes and metabolites associated with cognitive function as well as NAFLD development or prevention have been established in this study. Our data also suggest that BA receptor-regulated signaling likely has a role in regulating both liver and brain health.

\section{Materials and methods \\ Mice}

C57BL/6 wild-type (WT) male mice that were free of specific pathogens (Jackson Laboratory, Sacramento, CA, USA) were contained in steel microisolator cages with a 12-h light/dark cycle at $22^{\circ} \mathrm{C}$. After weaning, mice were fed with either a control diet (CD) containing 6.2\% fat, $44 \%$ carbohydrate, and $18 \%$ protein or an FPC diet constituting $29 \%$ fat, $34 \%$ sucrose, and $1.25 \%$ cholesterol (w/ w, TD. 160,785, Envigo, Indianapolis, IN, USA) plus 42 $\mathrm{g} / \mathrm{L}$ glucose and fructose $(55 \% / 45 \%, \mathrm{w} / \mathrm{w})$ in drinking water. At 3 months of age, FPC diet-fed mice were 
randomly assigned to two subgroups with or without inulin (6\%, Micro ingredients, Montclair, CA, USA) supplementation for 5 months. At 8 months of age, all three groups $(n \geq 4)$ of mice were euthanized. Experiments were conducted in accordance with the National Institutes of Health Guidelines for the Care and Use of Laboratory Animals under protocols approved by the Institutional Animal Care and Use Committee of the University of California, Davis.

\section{Histopathology and biochemical analysis}

Liver histology was analyzed by hematoxylin and eosin $(H \& E)$ stained section. Steatosis scores were graded on a scale of $0(<5 \%), 1(5-33 \%), 2(34-66 \%)$, and $3(>66 \%)$ based on the percentage of area that had fat [23]. Serum alanine aminotransferase (ALT) and alkaline phosphatase (ALP) (Pointe Scientific, Canton, MI, USA) levels were quantified per manufacturer protocols.

\section{Western blot analysis}

Liver and brain proteins $(40 \mu \mathrm{g})$ were subjected to SDSPAGE followed by transferring to PVDF membranes. The following primary antibodies (dilutions) were used: FASN (1:1000; Cell Signaling Technology, Danvers, MA, USA), CD36 (1:1000; Bioss Antibodies, Woburn, MA, USA), SCD1 (1:1000; Cell Signaling, Danvers, MA, USA), IL-17A (1:3000; eBiosciences, San Diego, CA, USA), TNFa (1; 1000; Bioss Antibodies, Woburn, MA, USA), FXR (1:250; Santa Cruz Biotechnology, CA, USA), HNF4a (1:1000; Aviva system biology, San Diego, CA, USA), SHP (Santacruz Biotechnology, Dallas, Texas, USA), PSD-95 (1:1000; Cell Signaling, Danvers, MA, USA), BDNF (1:1000; Millipore Sigma, St. Louis, MO, USA), TGR5 (1:3000; Lifespan Biosciences, WA, USA), and $\beta$-ACTIN (1:10000; Millipore Sigma, St. Louis, MO, USA). Horseradish peroxidaseconjugated secondary antibodies were then used to incubate the membranes. An ECL system with Pierce SuperSignal West Pico chemiluminescent substrates (Thermo Fisher Scientific, Rockford, IL, USA) was used to detect signals. Western blots protein band intensities were quantified by using ImageJ software (Version 1.53c) showed in the supplementary Figure 1.

\section{Gene expression profiling}

TRIzol (Invitrogen, Carlsbad, CA, USA) was used to isolate hepatic and microglial RNA followed by reverse transcription to generate cDNA. qRT-PCR was utilized on an ABI 7900HT Fast real-time PCR system using Power SYBR Green PCR Master Mix (Applied Biosystems, Foster City, CA, USA). Gapdh mRNA level was used to normalize mRNA levels. Primers used for qRTPCR listed in supplementary Table 1.

\section{Microglia isolation}

Brain microglia were isolated by enzymatical digestion using a neural tissue dissociation kit (Miltenyi Biotec, San Diego, CA) followed by magnetic-activated cell sorting with anti-CD11b coated magnetic beads (Miltenyi Biotec, Auburn, CA, USA).

\section{Long-term potentiation (LTP) study by electrophysiological recording}

Electrophysiological recordings were performed as previously described [23]. Briefly, coronal brain slices $(300 \mu \mathrm{m})$ were prepared and transferred to artificial cerebrospinal fluid (ACSF) described earlier [24]. After subsequent incubation for at least $1 \mathrm{~h}$ at room temperature, hemi-slices were transferred to the recording chamber, which was perfused with standard ACSF at a constant flow rate of $2 \mathrm{ml} / \mathrm{min}$. After stimulation of the schaffer collateral afferents, recording of field excitatory post-synaptic potentials (fEPSPs) were obtained from the stratum radiatum of the Cornu Ammonis (CA1) region in the hippocampus. Borosilicate capillaries with an outer diameter of $1.5 \mu \mathrm{m}$ (Sutter Instruments, Novato, CA, USA) and filled with $3 \mathrm{M} \mathrm{NaCl}$ (resistance, 1-2 M $\Omega$ ) were used to make extracellular recording electrodes. Baseline stimulation rate was $0.05-\mathrm{Hz}$. A Multiclamp 700B amplifier (Molecular Devices, Sunnyvale, CA) was used to filter the fEPSPs at $2 \mathrm{kHz}$ and digitized at $10 \mathrm{kHz}$. Collected data were analyzed with pClamp software version 10.3 (Molecular Devices, Inc., Sunnyvale, CA, USA). Slope values of fEPSPs were considered for quantitation of the responses. After 10 mins of stable baseline recording of fEPSPs was induced every 20 $\mathrm{s}$, LTP was prompted by high-frequency stimulation (HFS) comprising of a single train of $100-\mathrm{Hz}(1 \mathrm{~s})$ stimulation with the same intensity and pulse duration used in sampling of baseline fEPSPs.

\section{Open field behavior study}

Open field behavior tests for exploratory activity were recorded during the day light phase. The SmartCage ${ }^{\text {Tx }}$ system (AfaSci Inc., Redwood City, CA, USA) was used to monitor the spontaneous activity. Mice explored freely for $10 \mathrm{~min}$ and their activities were determined by infrared beam breaks ( $\mathrm{x}, \mathrm{y}$, and $\mathrm{z}$ photo-beam break counts) monitored and analyzed by Cage Center and CageScore 2 software, respectively (AfaSci Inc., Redwood City, CA, USA).

\section{$16 \mathrm{~S}$ rRNA gene sequencing}

Cecal DNA was extracted using ZR Fecal DNA Miniprep Kit (Zymo Research, Irvine, CA, USA). Barcoded $16 \mathrm{~S}$ rRNA gene amplicons of genomic DNA Illumina (San Diego, CA, USA) sequencing was performed based on published methods [25]. Variable region 4 of the $16 \mathrm{~S}$ rRNA gene was amplified and sequenced. A custom python based dbcAmplicons (https://github.com/msettles/ 
dbcAmplicons) was used for demultiplexing and classifying DNA sequence reads to identify and assign the reads by expected barcode in addition to primer sequences [25]. The Ribosomal Database Project Bayesian classifier was used to assign sequences to phylotypes [26]. Reads were assigned to the first Ribosomal Database Project taxonomic level with a bootstrap score $\geq 50$.

\section{Untargeted metabolomic study}

Cecal metabolites were quantified by gas chromatography time-of-flight mass spectrometry (GC-TOFMS) [27]. Acquired spectra were processed using the BinBase database, filtered, and matched against the Fiehn Mass Spectral Library of 1200 authentic metabolite spectra with retention index and mass spectrum information or against the NIST library. Pathway analyses were generated by MetaboAnalyst 3.0 [28]. Chemical similarity enrichment analysis was performed by ChemRICH [29].

\section{Bioinformatics and statistical analysis}

Volcano plots and Spearman's correlations were completed with $\mathrm{R}$ Statistical Software (The R Project for
Statistical Computing; https://www.r-project.org/). Data were expressed as means $\pm \mathrm{SD}$. Cecal metabolomics data were analyzed with ChemRICH [29] and MetaboAnalyst method [30]. Group differences in microbiota family level were calculated by Kruskal-Wallis test. All comparisons were calculated by two-tailed Student's $t$ test or one-way ANOVA followed by Tukey's test using GraphPad Prism 6 software (GraphPad Software, La Jolla, CA, USA). The $p$-values are adjusted for multiple comparisons using false discovery rate. A value of $p<0.05$ was considered statistically significant.

\section{Results}

Inulin prevents diet induced NAFLD

FPC diet intake for 7 months caused steatosis (Fig. 1a). FPC diet-fed mice had macrovesicular fat with grade 3 steatosis, and inulin-fed mice had improved fat scores (Fig. 1b). In addition, inulin-supplemented mice had normal liver weights and liver-to-body weight ratios (Fig. 1b). Moreover, FPC diet-fed mice had elevated ALT and ALP, and inulin supplementation reversed these changes (Fig. 1b).

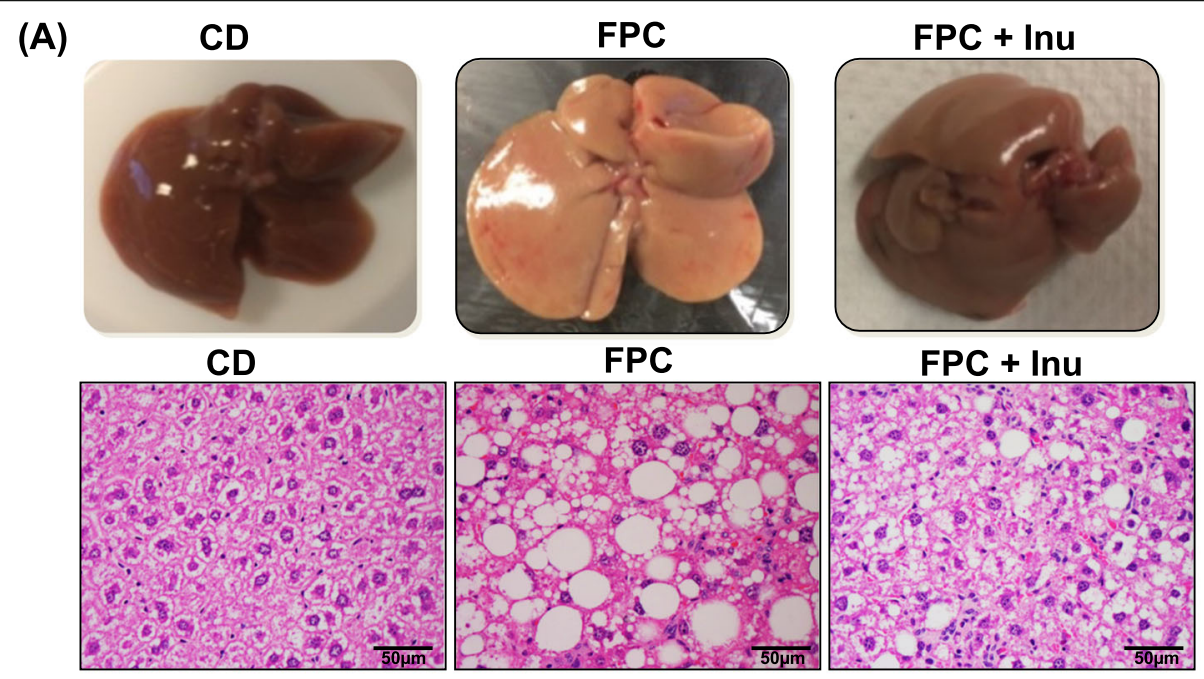

(B)

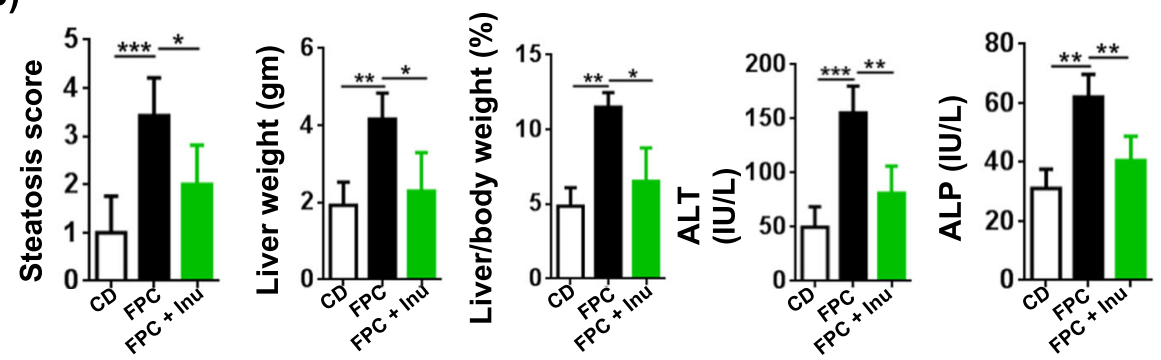

Fig. 1 Inulin supplementation prevents diet-induced fatty liver. C57BL6/J wild-type mice were fed with a healthy control diet (CD) or fat and sugar-enriched FPC diet since weaning (3 weeks old). At 3 months of age, FPC diet-fed mice were assigned into two subgroups, i.e., with or without inulin (Inu) supplementation for 5 months. All three groups of mice were euthanized when they were 8 months old. a Liver morphology and H\&E staining of the liver sections (scale bar represents $50 \mu \mathrm{m}$ ), b steatosis score, liver weight, liver-to-body weight ratio, serum ALT, and serum ALP. Data expressed as mean \pm SD. $n=4$ per group. ${ }^{*} p<0.05,{ }^{* *} p<0.01,{ }^{* *} p<0.001$ 
Inulin improves hepatic metabolism in FPC diet-fed mice FPC diet intake increased the expression of genes involved in fatty acid synthesis, uptake, and mobilization as evidenced by increased mRNA levels of Acetyl-CoA carboxylase 1 (Acc1), Stearoyl-CoA desaturase-1 (Scd1), Fatty acid synthase (Fasn), Cluster of differentiation 36 (Cd36), Fatty Acid Binding Protein 4 (Fabp4), and Sterol regulatory element-binding protein 1 (Srebp1c) as well as genes implicated in fatty acid oxidation such as Peroxisome proliferator-activated receptor $\alpha$ (Ppar $\alpha)$, PPAR $\alpha$-regulated Cyp4a14 and Cyp4a10, and Cyp2e1. In contrast, inulin supplementation prevented these changes in FPC diet-fed mice (Fig. 2a). Similarly, FPC diet increased protein levels of FASN, CD36, and SCD1, which were all reduced by inulin. Representative Western blots are shown in Fig. $2 \mathrm{~b}$.

Inulin prevents FPC diet-induced inflammatory signaling IL-17A is implicated in the development of chronic inflammatory diseases in the brain, skin, and liver as well as liver cancer [31-34]. FPC diet-fed mice had increased mRNA level of Roryt, a transcriptional factor for $\mathrm{Il}-17 \mathrm{ex}$ pression, accompanied by elevated hepatic protein levels of IL-17A and TNF $\alpha$ as well as mRNA levels of $I l 6, I l 1 \beta$, and $\operatorname{Tg} f \beta$, which generate Th17 cells; inulin supplementation prevented these changes (Fig. 3a, b). However, IL10 cytokine family member $I l 22$ displayed opposite trends in response to FPC diet intake and inulin supplementation (Fig. 3a). Other inflammatory and fibrosis-related genes such as Tnfo, Mcp-1, Col1a1, Timp1, Mmp2, and Mmp9 as well as macrophage or neutrophil genes such as $F 4 / 80$, $C d 11 b$, and $C d 68$ were induced by FPC diet intake and reduced by inulin (Fig. 3a).

\section{Inulin mitigates FPC-reduced hippocampal LTP and neuroplasticity}

FPC diet-fed mice had reduced LTP at Schaffer collateralCA1 synapses compared with that of CD-fed mice, and inulin-supplemented mice recovered such a reduction (Fig. 4a, b). In addition, open field behavior study revealed that FPC diet intake increased travel distance and central time spending, and inulin supplementation prevented such increases accompanied by increased rearing (Fig. 4c). Furthermore, FPC diet-fed mice had reduced postsynaptic density-95 (PSD-95), a potent regulator of synaptic strength, as well as brain derived neurotrophic factor (BDNF); however, inulin-supplementation prevented these negative effects caused by FPC intake (Fig. 4d) and improved synaptic plasticity.

\section{Inulin reverses FPC diet-induced microglia activation}

Microglia have pivotal roles in the inflammatory process in various neurodegenerative conditions; however, little

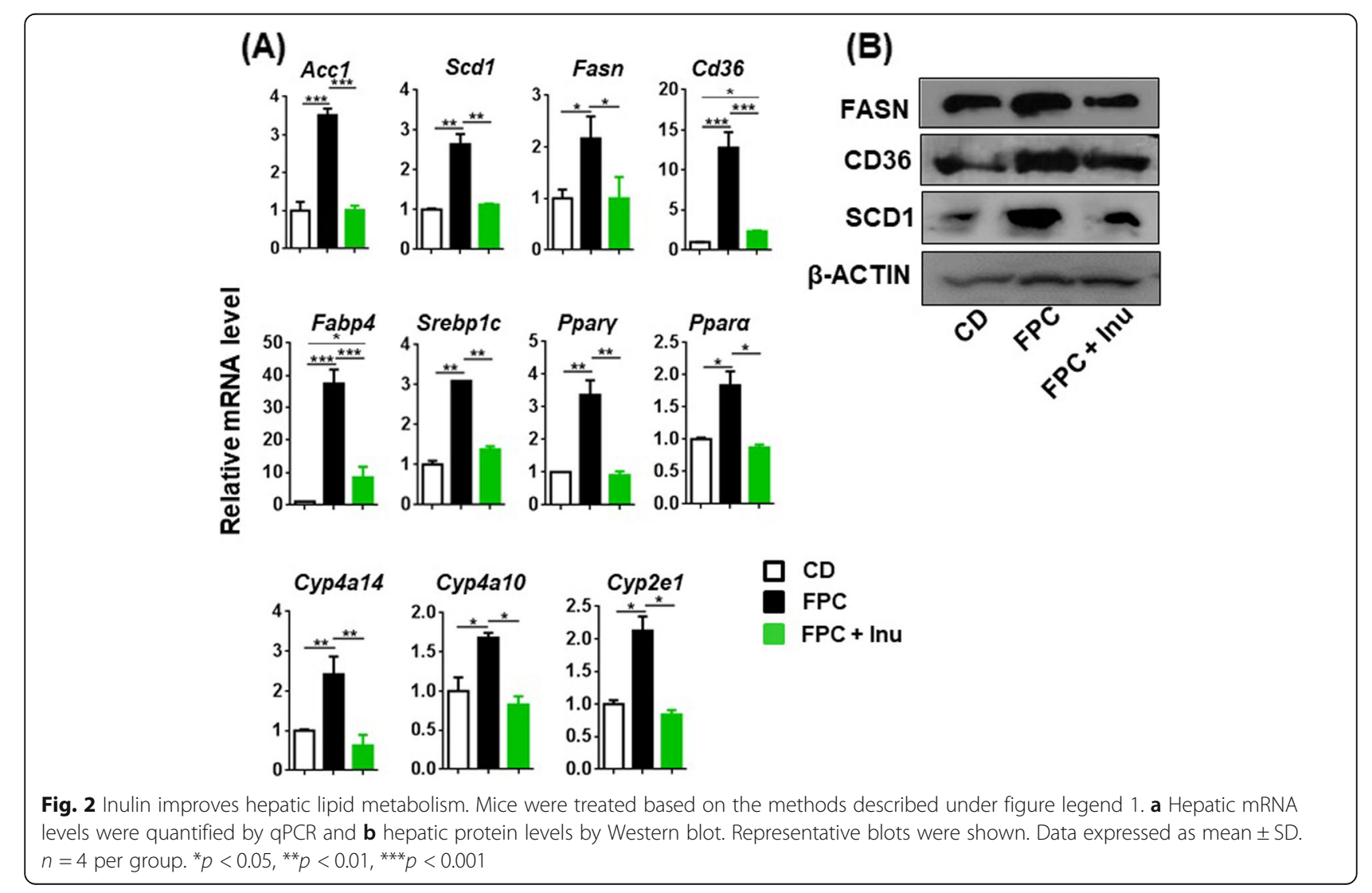



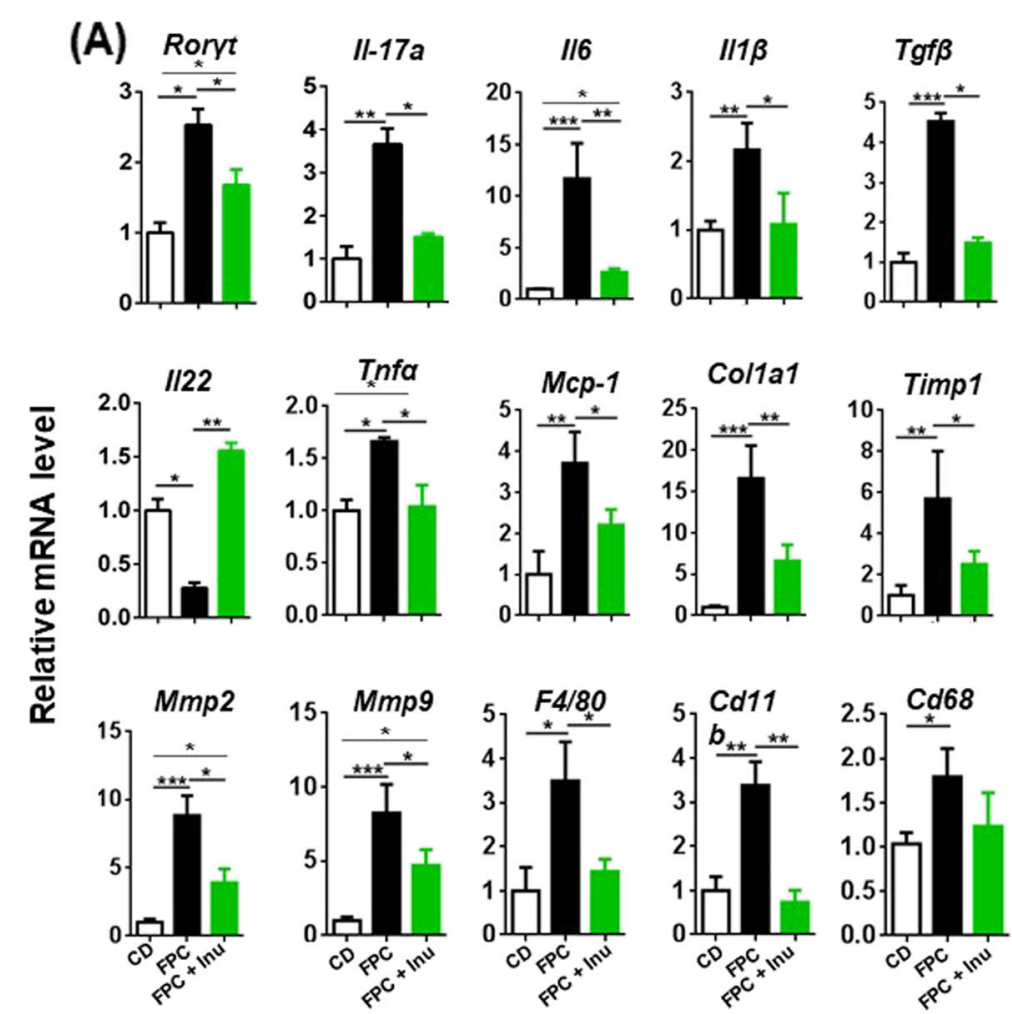

\section{(B)}

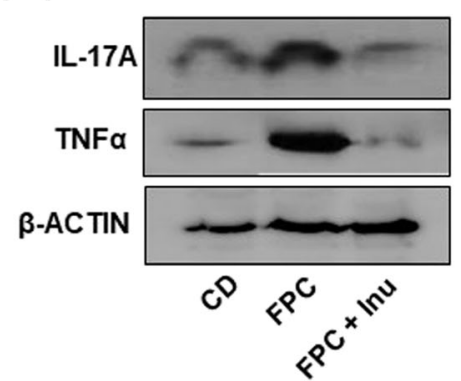

Fig. 3 The effect of FPC diet and inulin supplementation on hepatic inflammatory signaling. Mice were treated based on the methods described under figure legend 1. $\mathbf{a}$ Hepatic mRNA levels were quantified by $\mathrm{qPCR}$ and $\mathbf{b}$ hepatic protein levels by Western blot. Representative blots were shown. Data expressed as mean \pm SD. $n=4$ per group. ${ }^{*} p<0.05,{ }^{* *} p<0.01,{ }^{* * *} p<0.001$

is known about the effect of inulin on microglia activation. In this study, FPC diet-fed mice had elevated mRNA levels of Roryt, Il-17a, as well as $I l 22$ and increased protein levels of inflammatory marker IL-17A, IL6, and TNFa. (Fig. 5a, b). We showed that FPC diet intake increased the expressions of proinflammatory modulators Il1 $\beta$, Il6, Tnfo, and Nos 2 in the brain, and such inductions were reversed by inulin supplementation (Fig. 5a). Similar expression trends were noted for genes implicated in inflammation or markers for macrophage such as $\mathrm{Mcp}-1, \mathrm{Ccl}$, Ccl17, Ccl20, and Nos2 as well as F4/ 80 and $C \times 3 \mathrm{cr} 1$ (Fig. 5a). The mRNA levels of microglia calcium-activated potassium and voltage-dependent potassium channels $K c a 3.1$ and $K v 1.2$, which are involved in microglial activation and inflammation, were also elevated in FPC diet-fed mice and reversed by inulin supplementation (Fig. 5a). Together, inulin prevented FPC dietinduced neuroinflammation and microglia activation.

\section{FPC and inulin supplementation modulate intestinal microbiota}

Firmicutes and Bacteroidetes are the most abundant gut bacterial phyla. FPC diet-fed mice had reduced Firmicutes while inulin-supplemented mice had increased Bacteroidetes (Fig. 6a). FPC diet intake resulted in increased Proteobacteria, Actinobacteria, and Verrucomicrobia, and inulin intake only reduced the abundance of Actinobacteria (Fig. 6a). 

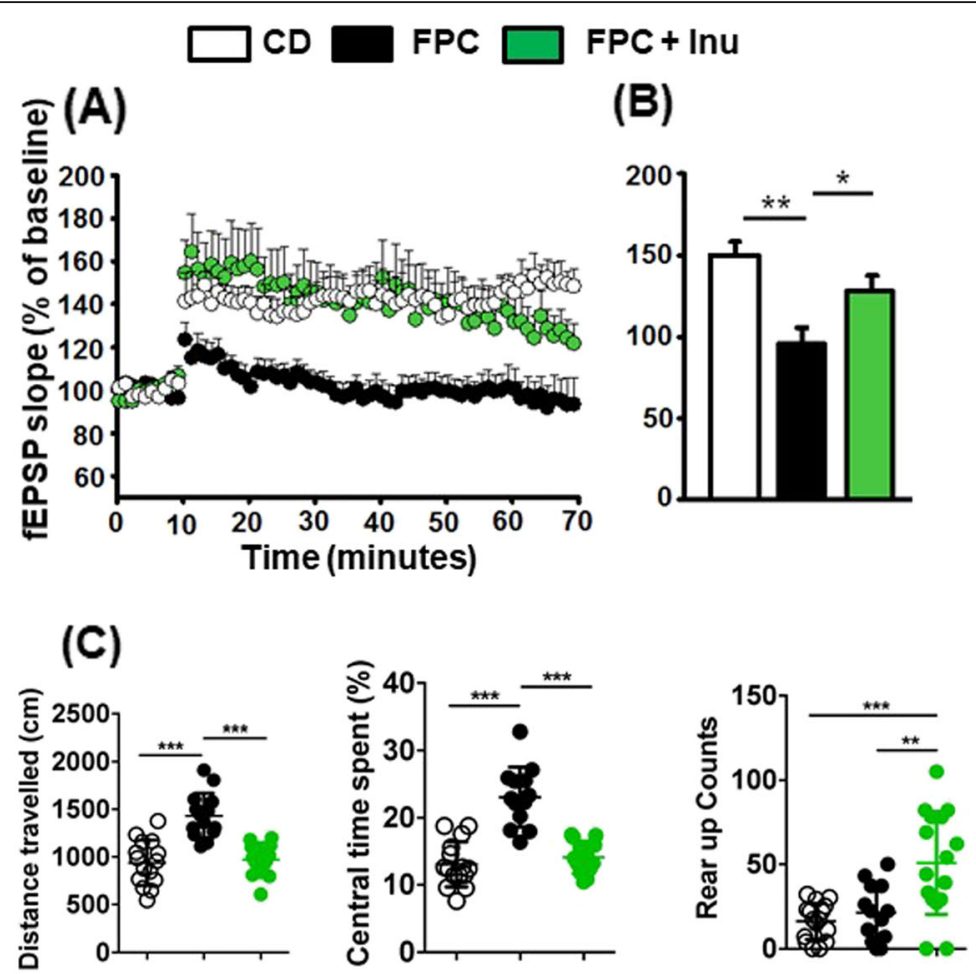

(D)

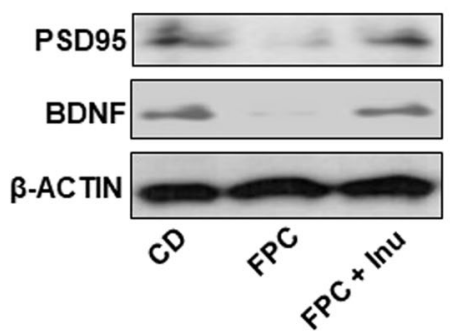

Fig. 4 FPC diet-induced synaptic impairment is ameliorated by inulin supplementation. a Scatter plot showing high-frequency stimulationinduced LTP, and $\mathbf{b}$ a bar graph showing LTP calculated by averaging the change in fEPSP slope apparent between 50 and 60 min after high frequency stimulation. All data are presented as the percentage change in fEPSP slope means \pm SEM from baseline, $\mathbf{c}$ Open field behavior study, d Western blot data reveal the protein level in brain homogenate. Data expressed as mean \pm SD. ( $n=3$ for A-B and $n=13-16$ for $C$, and $n=4$ for D). ${ }^{*} p<0.05,{ }^{* *} p<0.01$

The reduction of Firmicutes in FPC diet-fed mice is a direct result of the reduction in the families Lachnospiraceae and Lactobacillaceae, which promote gut health and fight inflammation; however, inulin supplementation increased their abundances (Fig. 6b) and reduced inflammation. In contrast, Erysipelotrichaceae, which is involved in cholesterol metabolism, as well as Peptostreptococcaceae, Clostridiaceae, and Eubacteriaceae genera under Firmicutes were enriched by FPC diet, however inulin reduced them (Fig. 6b). Moreover, inulin-fed mice had an increased family under Bacteroidetes, i.e., Porphyromonadaceae, which protects the gut from infection in mouse models [35]
(Fig. 6b). The family Helicobacteraceae under Proteobacteria, which is implicated in gastrointestinal inflammation [36], increased in FPC diet-fed mice, and inulin supplementation reduced it (Fig. 6b). Additionally, increased Proteobacteria in FPC diet-fed mice could be attributed to increased Sutterellaceae, which is implicated in gut inflammation as well as autism and Down syndrome [37, 38]. Under Actinobacteria phylum, Coriobacteriaceae, which has a role in cholesterol metabolism, increased with FPC diet intake and reduced with inulin supplementation (Fig. 6b).

At the genus level, FPC diet-fed mice had increased levels of Allobaculum and Holdemanella (Erysipelotrichaceae 
(A)
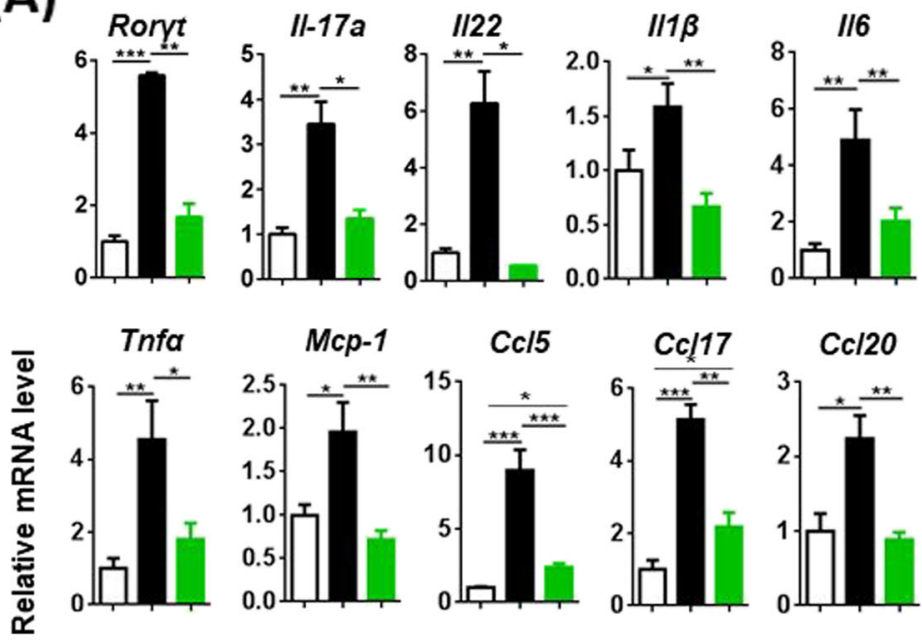

Cc/20
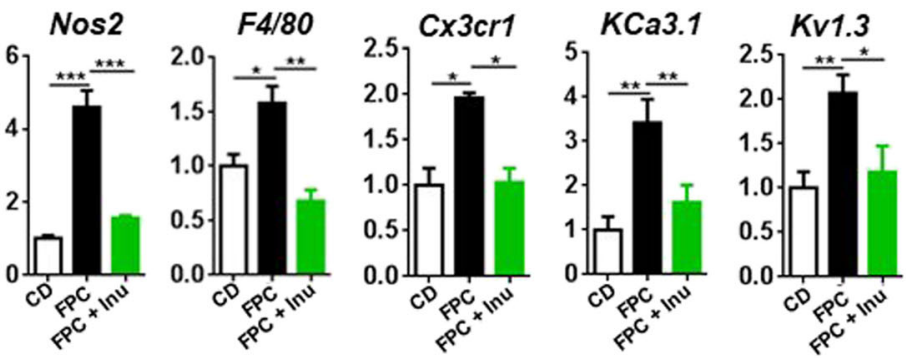

(B)

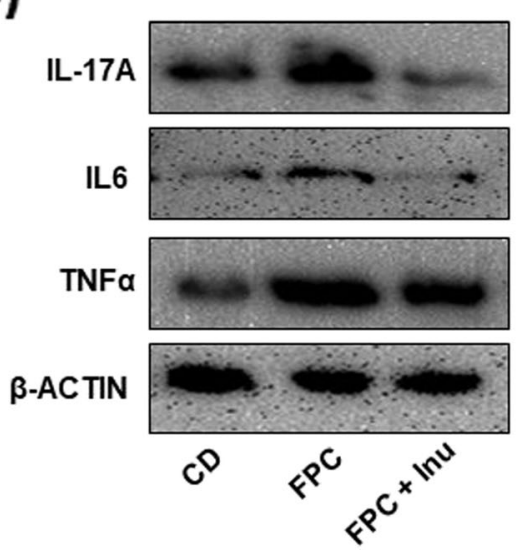

Fig. 5 The effects of diet and inulin supplementation on brain inflammation. Mice were treated based on the methods described under figure legend 1. a Microglia mRNA level and $\mathbf{b}$ brain protein level representative image was shown. Data expressed as mean \pm SD. $n=4$ per group. ${ }^{*} p<0.05,{ }^{* *} p<0.01,{ }^{* * *} p<0.001$

family), Romboutsia (Peptostreptococcaceae family), Romboutsia (Peptostreptococcaceae family), Olsenella and Clostridium XVIII (Coriobacteriaceae family), Lactonifactor (Clostridiaceae family), Eubacterium (Eubacteriaceae family), Enterococcus (Enterococcaceae family), Parasutterella (Sutterellaceae family), and Dorea and Acetatifactor (Lachnospiraceae family); inulin supplementation resulted in reductions in these genus except for Acetatifactor (Fig. 6c). In contrast,
FPC diet reduced Lactobacillus (Lactobacillaceae family), Eisenbergiella (Lachnospiraceae family), Butyrivibrio (Lachnospiraceae family), Clostridium XIVa (Clostridiaceae family), and Bifidobacterium (Bifidobacteriaceae family), and inulin supplementation resulted in their increases except for Bifidobacterium. Under the family Porphyromonadaceae, inulin enriched Barnesiella and Coprobacter (Fig. 6c). 

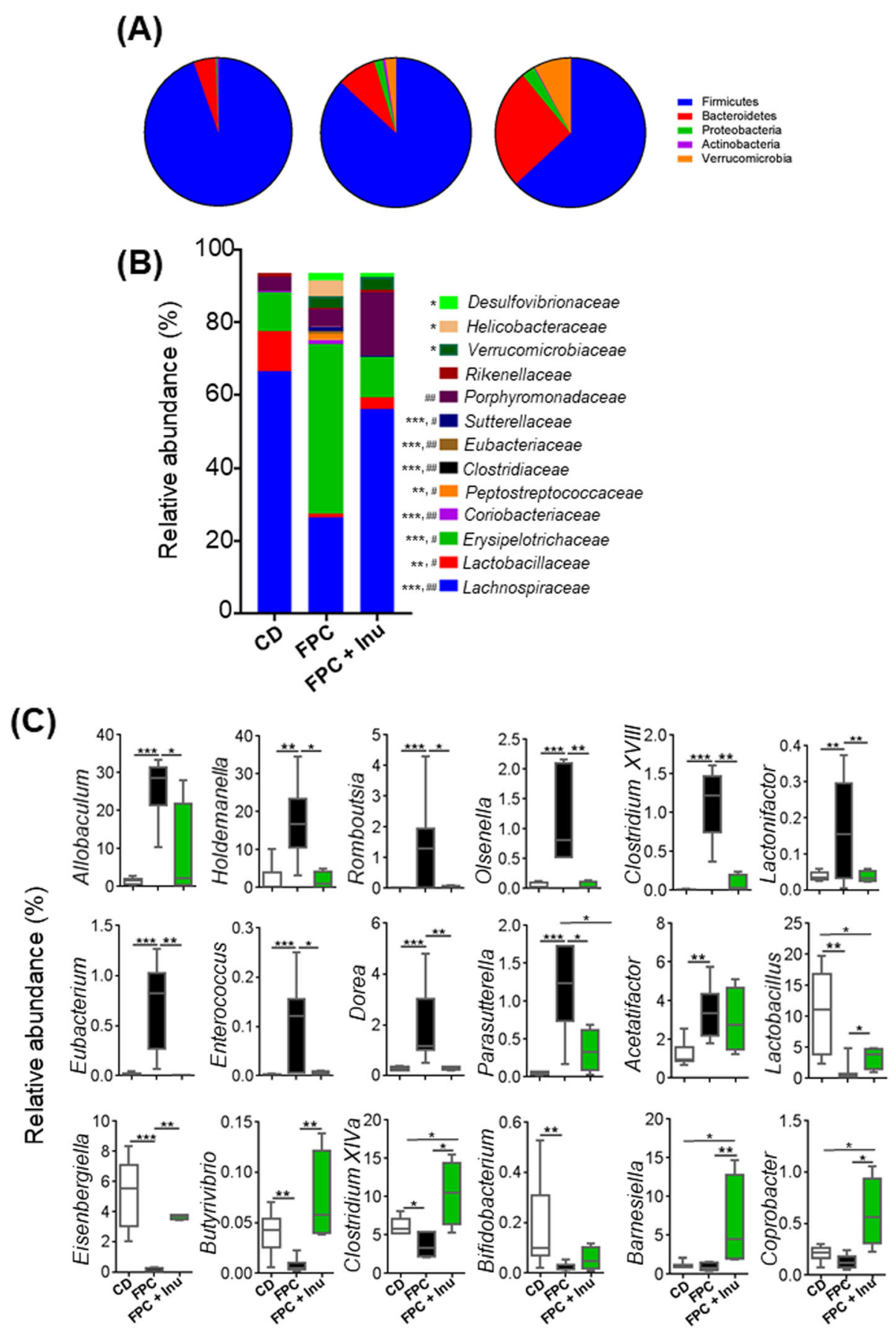

Fig. 6 The effects of diet and inulin supplementation on the gut microbiota. Mice were treated based on the methods described under figure legend 1. Relative abundance of cecal microbiota of a phylum, $\mathbf{b}$ family (*, WT vs. FPC; \#, FPC vs. FPC + Inulin), and $\mathbf{c}$ genus levels. Data are expressed as means \pm SD ( $n=8$ for CD-fed mice, $n=7$ for FPC-fed mice, and $n=4$ for inulin-supplemented FPC-fed mice). ${ }^{*} p<0.05$,

${ }^{* *} p<0.01,{ }^{* * *} p<0.001$

Inulin ameliorates diet-induced dysregulated BA signaling BA synthesis is jointly regulated by host and gut microbes. Dysregulated BA synthesis is implicated in hepatic inflammation and neurological diseases [24, 39]. We therefore studied the expression of genes that regulate BA homeostasis. FPC diet-fed mice had reduced mRNA and protein levels of FXR and its target gene small heterodimer partner $(S h p)$ as well as its encoded protein, which were reversed by inulin supplementation (Fig. 7a, b). In consistency, FXR-regulated genes that are responsible for BA synthesis such as hepatic Cyp $7 b 1$ and Cyp27a1 had the same expression patterns (Fig. 7a). 

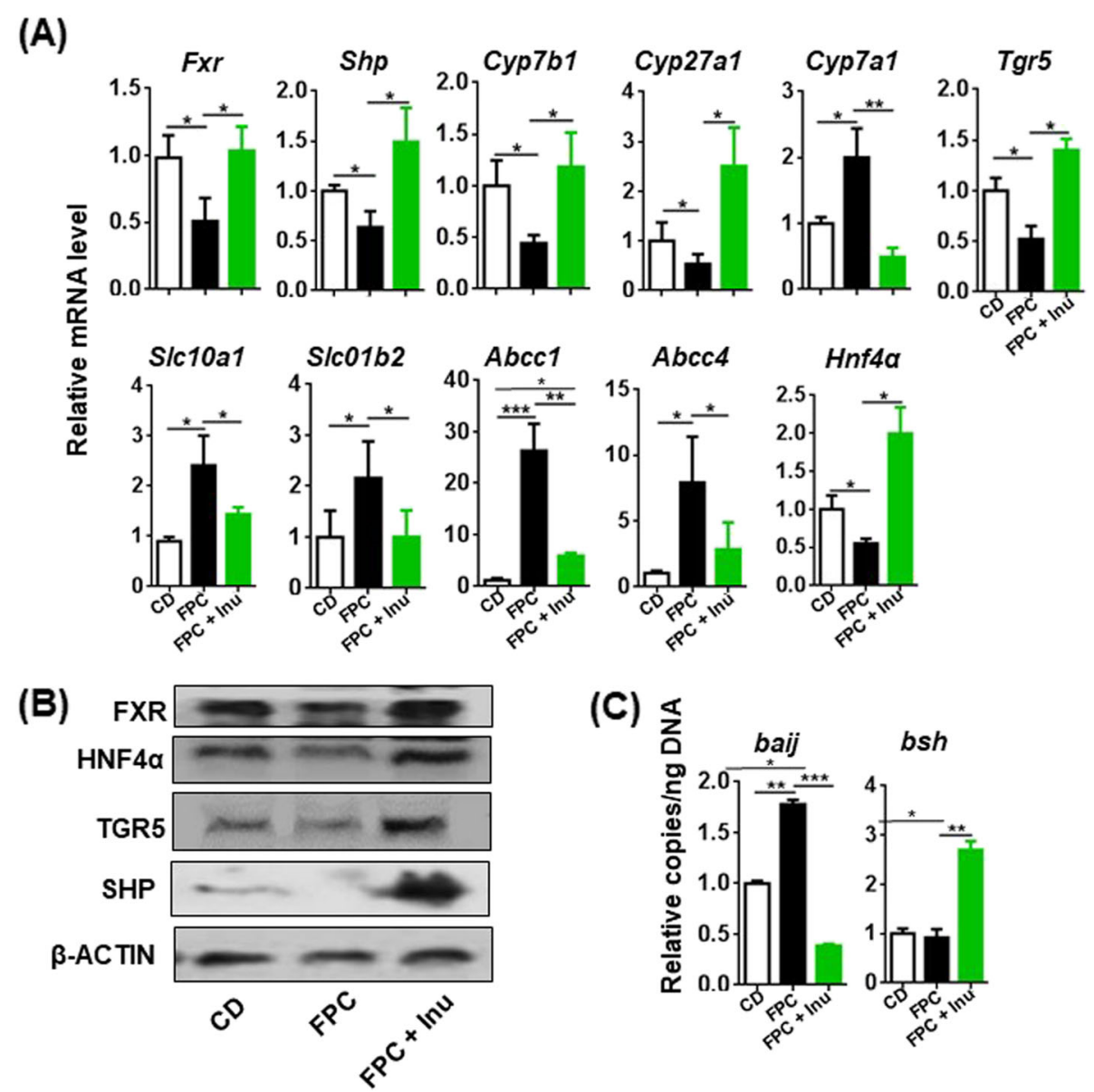

(C)

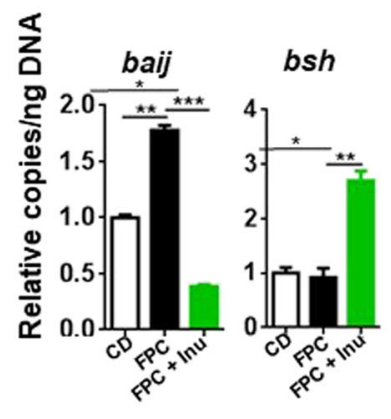

Fig. 7 The effect of diet and inulin on expression of genes implicated in bile acid homeostasis. Mice were treated based on the methods described under figure legend 1. a Relative level of hepatic mRNA was quantified by qPCR, $\mathbf{b}$ hepatic protein expression level by Western blot, and $\mathbf{c}$ the copy number of fecal bacteria genes that generate BAs was quantified by qRT-PCR. (bail, bile acid inducible operon gene of 7ahydroxylase; bsh, bile salt hydrolase), Data expressed as mean \pm SD. $n=4$ per group. ${ }^{*} p<0.05,{ }^{* *} p<0.01,{ }^{* * *} p<0.001$

Additionally, the level of Cyp7a1, a rate-limiting enzyme for BA synthesis, was increased in the steatotic livers but reduced by inulin supplementation (Fig. 7a). Moreover, the mRNA and protein levels of hepatic TGR5, another FXR target gene, were reduced in FPC diet-fed mouse livers but increased with inulin supplementation (Fig. 7a, b). Further, BA uptake genes Slc10a1 and Slc01b2 as well as transporter genes $A b b c 1$ and $A b b c 4$ were induced in FPC diet-fed mice, and inulin supplementation reversed these inductions, suggesting normalization of BA homeostasis (Fig. 7a). Lastly, hepatic HNF4 $\alpha$ was reduced by FPC diet intake and increased by inulin supplementation, which likely contributes to normalizing the hepatic transcriptional machinery (Fig. 7a, b). Together, diet-induced metabolic overburden inactivates FXR signaling, and inulin normalizes those changes.

The copy number of bacterial genes that produce BAs was also quantified using cecal DNA. FPC diet-fed mice had increased BA inducible operon $J$ (bai), a gene involved in BA $7 \alpha$-dehydroxylation, and inulin supplementation reduced it (Fig. 7c). However, the abundance of bile salt hydrolase gene ( $b s h$ ), which hydrolyzes conjugated BAs into free BAs, was unchanged by FPC diet intake but was increased by inulin (Fig. 7c).

TGR5 is abundantly expressed in the macrophage [40, 41]. We thus studied TGR5-regulated signaling in freshly isolated microglia. In consistency with the data generated from the digestive tract, FPC diet-fed mice had reduced Tgr 5 as well as TGR5-regulated Nos1 and Dio2 (Figure S2). The expressions of FXR and its target gene $\operatorname{Sh} p$ were also reduced in the microglia of FPC-fed mice, and inulin supplementation increased their expression levels (Figure S2). Thus, diet and inulin have an impact on BA-regulated signaling at the systemic level.

The effect of FPC diet and inulin on gut metabolites Metabolomics profiling was performed using cecum content by GC-TOF-MS, which identified 273 known 
metabolites. As per sparse partial-least-squares discriminant analysis (sPLS-DA), metabolites had different clusters (Figure S3). Pathway analysis revealed that the most significant difference between CD- and FPC diet-fed mice occurred in lipid metabolism that includes steroid hormone, BA, steroid, and fatty acid biosynthesis (Supplementary Table 2, Figure S3). However, inulin supplementation markedly changed sucrose metabolism in addition to amino acid and carbohydrate metabolism pathways, arginine, proline, butanoate, and starch. The top 15 most significantly changed pathways in response to FPC diet intake and inulin supplementation are listed in Supplementary Table 2 and Figure S3.

Fold changes in the cecal metabolites are shown in the volcano plots (Fig. 8a). Zymosterol and 2, 8-dihydroxyquinoline (2, 8-DHQ) are commonly found in both FPC vs. CD and FPC + inulin vs. FPC plots. Zymosterol, the precursor of cholesterol, was increased in FPC diet-fed mice but was reduced by inulin intake. In contrast, 2, 8-DHQ was markedly reduced in FPC diet-fed mice, and inulin supplementation increased it (Fig. 8a). Additionally, glucose 6-phosphate, fructose 6-phosphate, galactose 6-phosphate, xylose, and lyxose had reduced concentrations in FPC diet-fed mice (Fig. 8a). 3-Hydroxyphenylacetic acid, a metabolite of the flavonoid rutin which protects against glucose intolerance and obesity, was decreased due to FPC diet intake. In contrast, tocopherols $(\beta, \gamma, \delta)$ and digalacturonic acid were increased by FPC diet intake (Fig. 8a). Supplementation of inulin increased trans-4-hydroxyproline, $\beta$-sitosterol, isothreonic acid, and adenine (Fig. 8a).

Chemical similarity enrichment analysis shows that FPC diet-fed mice had reduced hexose phosphates, purine nucleosides, pyrimidinones, and sugar alcohols, which were increased by inulin supplementation (Fig. 8b). In contrast, cholestanes, a cholesterol metabolite, was increased in FPC diet-fed mice, and inulin supplementation reduced it (Fig. 8b). In addition, butyrate, a major SCFA produced by fermentation of inulin, was decreased by FPC diet intake and increased by inulin supplementation (Fig. 8b).

\section{The relationships between metabolites and the gut microbiota}

Among the 273 identified metabolites, 104 had significant changes in their scaled intensity due to FPC diet intake or inulin supplementation. These metabolites and 59 bacterial genera found in the mouse cecum were subjected to Spearman's correlation analysis (Fig. 9).

Genus Allobaculum, which was increased in FPC dietfed mice, was positively associated with cholesterol and zymosterol, but negatively correlated with sugar alcohols (xylose, and lyxose); glycolysis pathway metabolites (glucose-6-phosphate and fructose-6-phosphate); and pinitol, which has a role in regulating insulin function
(Fig. 9). In addition, genus Holdemanella was positively correlated with the scaled intensity of cecum fatty acids stearic acid and palmitic acid as well as fatty acid byproduct monostearin (Fig. 9). Genus Barnesiella, Coprobacter, Clostridium XIVa, and Butyrivibrio, which are negatively correlated with tocopherol, zymosterol, and monostearin, had increased abundances in inulin-fed mice (Fig. 9). Moreover, Lactobacillus and Eisenbergiella genus, which also increased due to inulin supplementation, were negatively associated with cholesterol, zymosterol, and fatty acids D-erythrosphingosine, palmitic acid, stearic acid, as well as arachidonic acid (Fig. 9). These fatty acids are clustered together and positively associated with the bacteria that were enriched in FPC diet-fed mice (Fig. 9).

\section{Discussion}

Our data revealed the detrimental effects of a WD, which simultaneously affects brain and liver health. Gut microbiota and their metabolites, which includes BAs, contributed to such an impact. It was also revealed through our data that inulin can reverse the pathological and molecular changes even while mice continue to consume a WD. Inulin reduced lipogenic gene such as Fasn, Cd36, Srebp1c, and Ppary. It also induced the expression of genes responsible for fatty acid oxidation and mobilization. Those metabolic benefits likely contribute to improved liver and brain phenotypes.

Recent study has shown that inulin induced cholestatic liver cancer in TLR5 knockout mice [19]. However, such a negative impact was not noted in the current study although long-term inulin supplementation was employed. These conflicting findings could be attributed to differences in animal models, diet, or environment, emphasizing the importance of precision nutritional supplementation [20]. However, both studies revealed the significant roles of BAs and the gut microbiota in liver disease development. Moreover, the current study suggests that hepatic metabolic status is an indicator for cognitive function or vice versa. Such a linkage is likely mediated through gut microbiota-driven inflammatory signaling.

IL-17, a cytokine produced by T helper 17 (Th17) cells plays a significant role in hepatic inflammation by inducing neutrophil infiltration and fatty acid release [42]. Increased IL-17A is also found in mouse models of Alzheimer's diseases and hepatic fibrosis [43, 44]. Moreover, our recent publication shows that short-term feeding of another WD leads to $\mathrm{T}$ helper type 1-/T helper type 17-biased skin inflammation before significant body weight gain is noted [31]. WD intake for 4 weeks promotes mild dermatitis and accumulation of IL-17A-producing $\gamma \delta \mathrm{T}$ cells in the skin. Furthermore, supplementation with cholestyramine, a BA sequestrant, can prevent skin inflammation along with a reduction in the infiltration of $\gamma \delta \mathrm{T}$ cells and the expression 

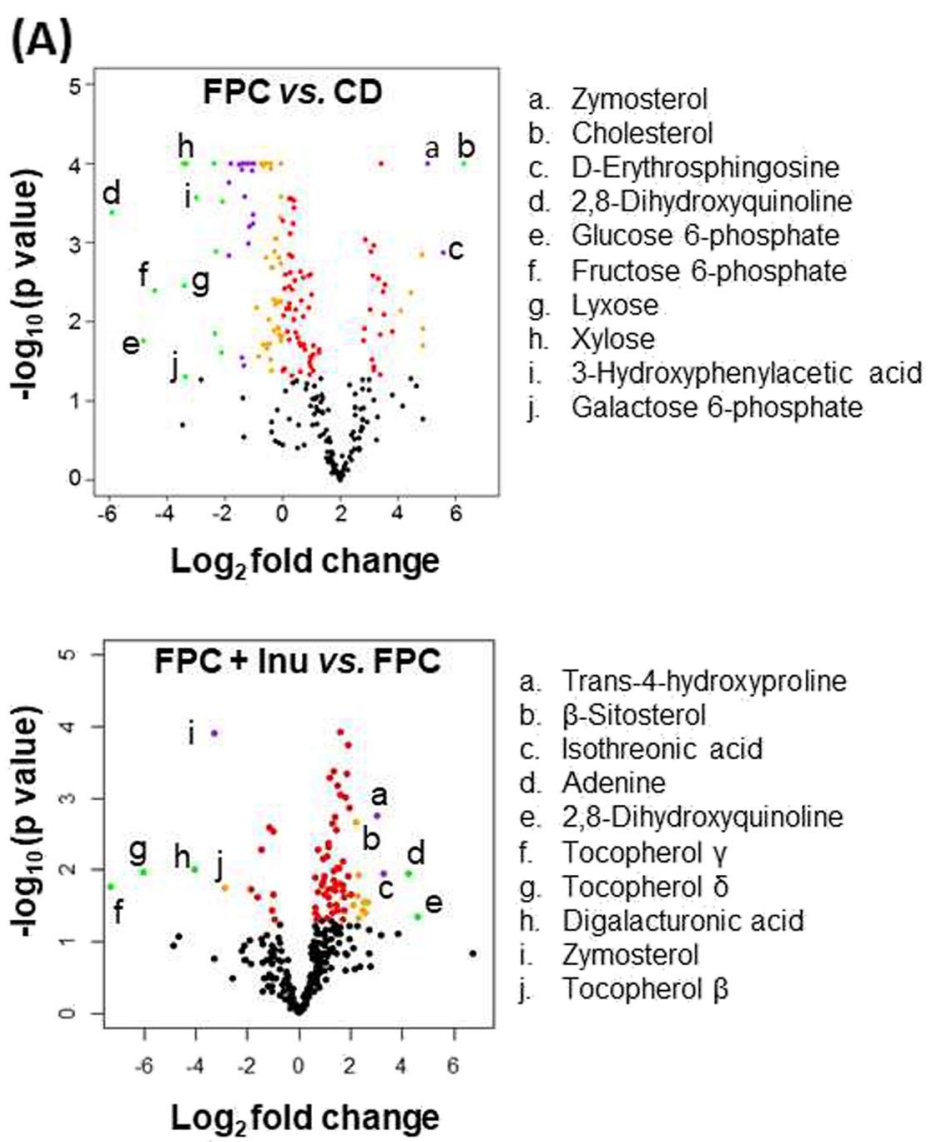

(B)

FPC vs. CD

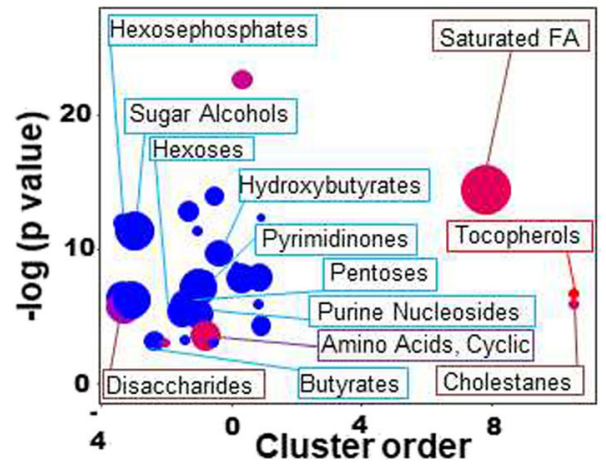

FPC + Inu vs. FPC

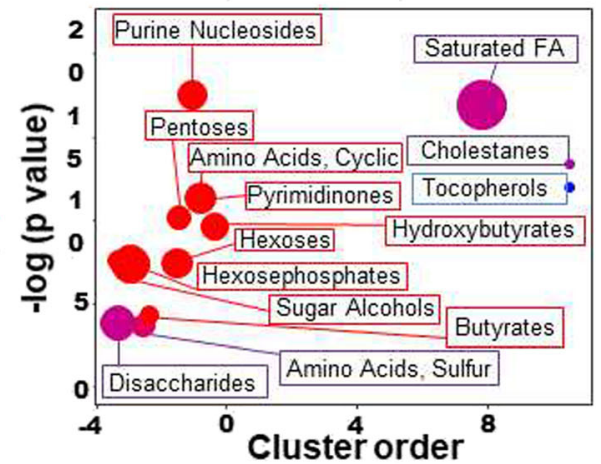

Fig. 8 Untargeted metabolomics study of cecal content. Mice were treated based on the methods described under figure legend 1. Volcano plots represent the cecal metabolomics profile between a FPC diet vs. CD and FPC diet + Inulin vs. FPC diet. The orange, purple, and green color represent the fold changes of 2,3, and 4, respectively with the $p$-value $<0.05$. b ChemRICH metabolite set enrichment statistics plot. The node color shows increased (red) or decreased (blue) metabolite sets in FPC diet vs. CD and FPC diet +Inulin vs. FPC diet. Only enrichment clusters are shown that are significantly different at $p<0.05$ (The node sizes represent the total number of metabolites in each cluster set)

of proinflammatory mediators [31]. The differentiation of Th17 cells relies on a variety of cytokines, and transcriptional factors such as ROR $\gamma$ t and TGF $\beta$ in synergy with IL6 are critical for generating Th17 cells [45]. Additionally, BAs have been found to be able to modulate RORyt signaling [46]. The simultaneous induction of microglial and hepatic
Roryt and $\mathrm{Il}-17 \mathrm{a}$ along with other inflammatory cytokines in FPC diet-fed mice, as well as their reductions in inulinsupplemented mice, suggest the significance of IL-17A in diet-associated inflammatory signaling at the systemic level.

A reduction in PSD-95 is associated with postsynaptic degeneration, altered synaptic plasticity, psychiatric 


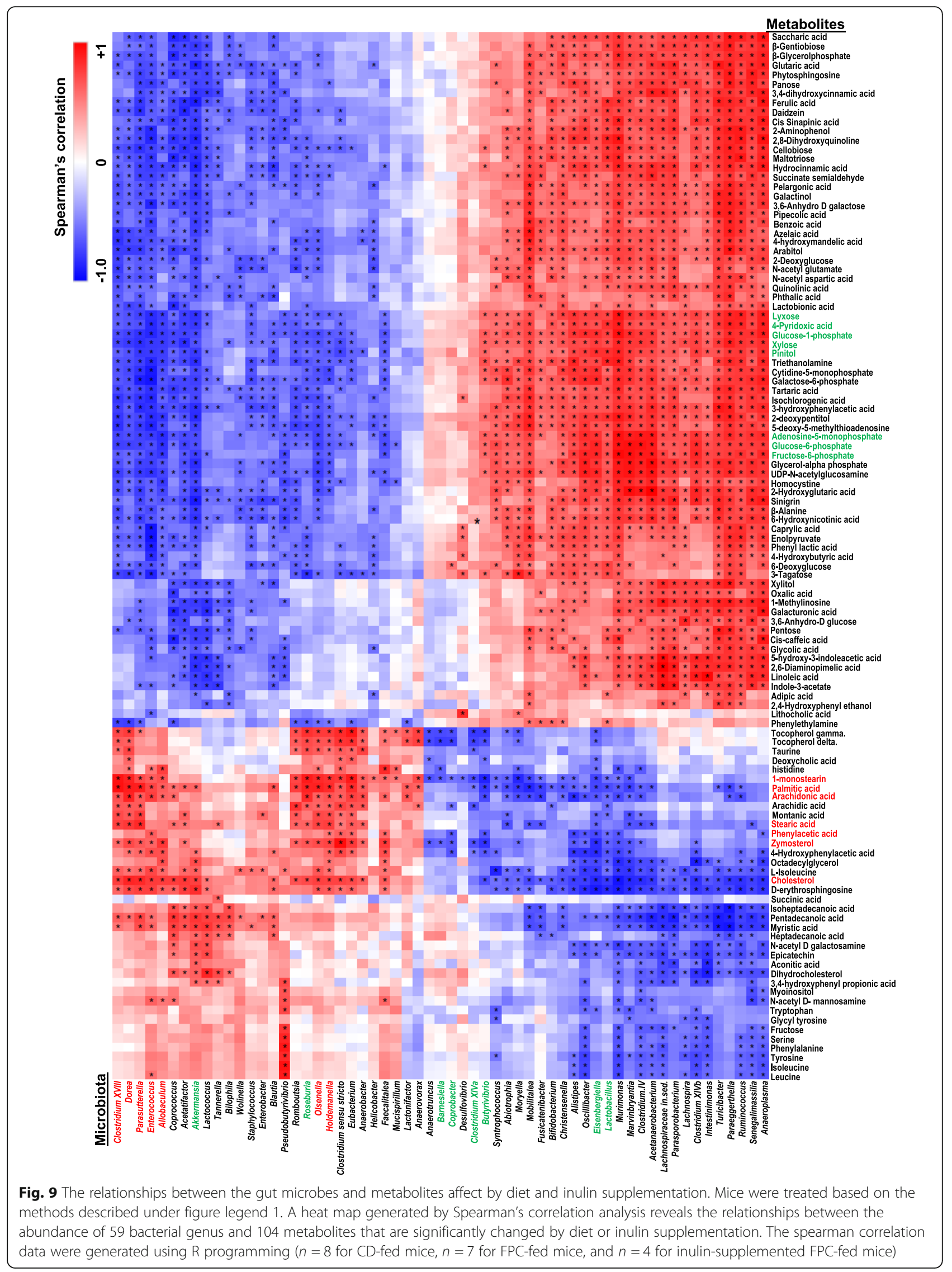


diseases, dementia, and Alzheimer's disease pathology [47]. BDNF is a vital neurotransmitter modulator required for memory and learning [48]. Our data revealed that FPC diet intake reduced LTP, BDNF, and PSD-5; however, inulin supplementation increased all of them. WD intake and obesity are associated with increased anxiety in humans and mice $[49,50]$. Here, we showed that inulin supplementation can modulate anxiety as well as exploratory behavior, revealing its beneficial effects. Together, the inflammatory signaling regulated by diet or inulin supplementation not only affects disease development but also has a significant impact on neuroplasticity and behavior.

Regarding the gut microbiota, FPC diet increased Erysipelotrichaceae and Coriobacteriaceae, which are associated with the dyslipidemia phenotypes found in obese individuals and animal models $[51,52]$. In contrast, inulin supplementation reduced Erysipelotrichaceae and Coriobacteriaceae, suggesting their roles in host lipid metabolism. It is likely that a diet rich in fat and cholesterol increased the abundances of Allobaculum, Holdemanella, and Olsenella, which obese mice and NAFLD patients also have increased abundance [53-55]. Our data revealed that inulin could prevent these changes along with reducing gut cholesterol and zymosterol. In consistency, APP/PS1 mouse model of Alzheimer's disease also had decreased Allobaculum [56]. Probiotic Lactobacillus has beneficial effects in improving insulin sensitivity as well as memory and cognition $[57,58]$. Our data showed that FPC diet intake reduced Lactobacillus and butyrate producing Butyrivibrio, and inulin supplementation increased their abundances. Barnesiella is an effective immunomodulator, which prohibits the colonization of pathogenic antibiotic-resistant bacteria in the gut [59]; Barnesiella was also increased in inulin-supplemented mice. Together, fermentable fiber inulin markedly shifted the gut microbiota as well as the metabolic phenotype, thereby generating health benefits.

BAs are generated by both host and microbial enzymes via metabolizing cholesterol. BA receptor FXR is pivotal for regulating metabolism and inflammation. In consistency, FXR knockout mice spontaneously develop NASH and liver cancer [60]. Our data suggest that FPC diet reduced FXR and FXR-regulated downstream effects. Additionally, HNF4 $\alpha$, a master regulator for hepatic gene expression, is also down regulated in FPC-fed mice. These compromised signaling pathways may account for NAFLD development. However, inulin supplementation restored them. In addition to regulating hepatic metabolism, FXR has a role in altering motor activity, cognitive function, and mood [10]. FXR agonist is currently in clinical trials to treat metabolic liver diseases [61]. Whether FXR agonists can be used to improve neuroplasticity warrants further investigation.
In addition to FXR, systemic inflammation is also accompanied by reduced TGR5 signaling. In consistency, the neuroprotective effect of TGR5 has also been shown in A $\beta 1-42$-induced cognitive impairment mouse models [12]. Additionally, TGR5 activation prohibits hepatic encephalopathy $[11,62]$. In the current study, the neuroprotective effect of TGR5 was further supported by the findings that inulin-improved neuroplasticity was accompanied by increased TGR-5-regualted signaling genes such as Dio 2 and Nos1 in the microglia. Thus, via improving metabolism and reducing inflammation, TGR5 can be a potential target for increasing neuroplasticity as well.

Metabolomics data showed that 2, 8-DHQ, a gut microbegenerated metabolite, was markedly reduced by FPC but increased by inulin. 2, 8-DHQ is a species-specific aryl hydrocarbon receptor agonist and has known benefits in reducing glucose intolerance and fighting obesity [63]. Additionally, glucose 6-phosphate, fructose 6-phosphate, galactose 6-phosphate, and sugar alcohols xylose and lyxose were reduced due to FPC diet intake. Conversely, inulin supplementation increased the abundance of sugar alcohols, which have lower caloric value, clearly indicating increased fermentation. Moreover, inulin increased the production of butyrate, which can be an energy source for gut epithelial cell renewal. ChemRICH analysis showed FPC diet-fed mice had decreased hexose phosphates, purine nucleosides, and pyrimidinones, which were all increased with inulin supplementation. Hexose phosphate molecules are key molecules in the pentose phosphate pathway which generates NADPH used in fatty acid biosynthesis [64]. Moreover, inulin-supplemented mice also had increased trans-4-hydroxyproline, which is implicated in wound healing [65] and $\beta$-Sitosterol, which boosts immunity [66]. Furthermore, isothreonic acid, a metabolite of vitamin C, and adenine, known as vitamin B4, were all elevated in the cecum of inulin-fed mice.

Metabolites such as palmitic acid are increased in neurodegenerative diseases such as Parkinson's disease and Alzheimer's disease [67]. Certain fatty acids such as monostearin, palmitic acid, arachidonic acid (phospholipids), stearic acid, zymosterol, and D-Erythrosphingosine, which clustered together, were positively correlated with FPC diet-increased microbiota Dorea, Parasutterella, Enterococcus, Allobaculum, Olsenella, and Holdemanella and negatively associated with inulin-increased Lactobacillus and Eisenbergiella. Thus, gut microbes likely altered the production of neurotransmitter-related metabolites and affected neural function.

\section{Conclusions}

Our results indicate that, chronic consumption of FPC diet negatively impact liver health and cognition. Supplementing fermentable fiber inulin into FPC diet reduced the impact of FPC diet and improved cognitive health. 
Taken together, diet-associated gut microbes and their derived metabolites through inflammatory signaling such as IL-17A and bile acid receptor signaling concomitantly affect the health of the liver and brain.

\section{Supplementary Information}

The online version contains supplementary material available at https://doi. org/10.1186/s40364-020-00239-8.

\section{Additional file 1: Table S1. Primers used for qPCR. Table S2.}

Metabolic Pathways and Function Analysis.

Additional file 2: Figure S1. Quantification of western blots showed in Figs. 2b, 3b, 4d, 5b and 7b. (A) Relative protein level of hepatic lipid biosynthesis and metabolism pathway, (B) relative protein level of hepatic inflammation, (C) relative protein level of brain homogenate, (D) relative protein level of brain inflammation, and (E) relative protein level of hepatic bile acid metabolism and synthesis. Data expressed as mean \pm SD. $n=4$ per group. ${ }^{*} p<0.05,{ }^{* *} p<0.01,{ }^{* * *} p<0.001$.

Additional file 3: Figure S2. The effect of diet and inulin on microglia bile acid signaling. Microglia were freshly isolated to extract RNA. The mRNA levels were quantified by qPCR. Data expressed as mean \pm SD. $n=$ 4 per group. ${ }^{*} p<0.05,{ }^{* *} p<0.01,{ }^{* * *} p<0.001$.

Additional file 4: Figure S3. The effect of diet and inulin on metabolomics profile. Untargeted metabolomics profile of cecal sample of CD-fed, FPC-fed, and FPC-fed with inulin supplemented mice. (A) sPLSDA based analysis of cecal metabolomics, (B) Pathway enrichment analysis of FPC vs. CD fed and FPC + Inu fed vs. FPC fed mice based on small molecule pathway database, (C) Pathway analysis of FPC vs. CD fed and FPC + Inu. fed vs. FPC fed mice based on KEGG database, (D) Heatmap of cecal metabolites clustered with Pearson and Wald statistical analysis ( $n=8$ for CD-fed mice, $n=7$ for FPC-fed mice, and $n=4$ for inulinsupplemented FPC-fed mice).

\section{Abbreviations}

FPC: Fructose, palmitate, and cholesterol; CD: Control diet; WD: Western diet; H\&E: Hematoxylin and eosin; NAFLD: Non-alcoholic fatty liver disease; NASH: Non-alcoholic steatohepatitis; PVDF: Polyvinylidene difluoride; CA: Cornu ammonis; ALT: Alanine transaminase; ALP: Alkaline phosphatase; BA: Bile acid; FXR: Farnesoid x receptor; TGR5: Takeda G protein-coupled receptor 5; LTP: Long-term potentiation; ACC: Acetyl CoA carboxylase; FASN: Fatty acid synthase; fEPSP: Field excitatory postsynaptic potentials; HCC: Hepatocellular carcinoma; TLR: Toll-like receptor; 2, 8-DHQ: 2, 8dihydroxyquinoline; AHR: Aryl hydrocarbon receptor; ACC1: Acetyl-CoA carboxylase 1; SCD1: Stearoyl-CoA desaturase-1; Cd36: Cluster of differentiation 36; Fabp4: Fatty acid binding protein 4; Srebp1c: Sterol regulatory element-binding protein 1; Ppara: Peroxisome proliferatoractivated receptor $a$; Bdnf: Brain derived neurotrophic factor; PSD95: Postsynaptic density-95; HFD: High-fat diet; SCFA: Short-chain fatty acid; DCA: Deoxycholic acid

\section{Acknowledgements}

Not applicable.

\section{Authors' contributions}

YJW designed the study; PKJ, LS, JDL, YH, and MN performed the experiments, PKJ, LS, MN, JDL, YH, YL, LWJ, IM, and YJW analyzed and interpreted the data; PKJ, LS, MN, and YJW wrote the manuscript; all authors commented and approved the final manuscript.

\section{Funding}

This study is supported by grants funded by National Institutes of Health U01CA179582 and R01CA222490, California Department of Public health for Alzheimer's Disease Research Grant H 18-10925-0, and Mouse Metabolic Phenotyping Center (MMPC) Microbiome Program (30835-60), Subaward A19-2654.

\section{Availability of data and materials}

All data generated or analyzed during this study are included in this article.

Ethics approval and consent to participate

This study is approved by institutional animal cure and use committee of University of California Davis.

\section{Consent for publication}

Informed consent form for publication was obtained from all authors.

\section{Competing interests}

The authors state no competing of interest.

\section{Author details}

${ }^{1}$ Department of Medical Pathology and Laboratory Medicine, University of California, Davis Health, Room 3400B, Research Building III, 4645 2nd Ave, Sacramento, CA 95817, USA. ${ }^{2}$ Department of Pediatrics, Cedars Sinai Medical Center, Los Angeles, CA 90048, USA. ${ }^{3}$ Institute of Interdisciplinary Integrative Medicine Research, Shanghai University of Traditional Chinese Medicine, Shanghai 201203, China. ${ }^{4}$ Department of Gastroenterology, Zhujiang Hospital, Southern Medical University, Guangzhou 510282, China. ${ }^{5}$ Department of Infectious Diseases, Nanhai Hospital, Southern Medical University, Foshan 528200, China.

Received: 20 August 2020 Accepted: 21 October 2020

Published online: 06 November 2020

\section{References}

1. Seo SW, Gottesman RF, Clark JM, Hernaez R, Chang Y, Kim C, Ha KH, Guallar E, Lazo M. Nonalcoholic fatty liver disease is associated with cognitive function in adults. Neurology. 2016;86(12):1136-42.

2. Yaffe K, Kanaya A, Lindquist K, Simonsick EM, Harris T, Shorr RI, Tylavsky FA, Newman AB. The metabolic syndrome, inflammation, and risk of cognitive decline. Jama. 2004;292(18):2237-42.

3. Noble EE, Hsu TM, Liang J, Kanoski SE. Early-life sugar consumption has long-term negative effects on memory function in male rats. Nutr Neurosci. 2019:22(4):273-83.

4. Jena PK, Sheng L, Liu HX, Kalanetra KM, Mirsoian A, Murphy WJ, French SW, Krishnan W, Mills DA, Wan YY. Western diet-induced Dysbiosis in Farnesoid $X$ receptor knockout mice causes persistent hepatic inflammation after antibiotic treatment. Am J Pathol. 2017;187(8):1800-13.

5. Wan Y-JY, Sheng L. Regulation of bile acid receptor activity. Liver Res. 2018; 2(4):180-5.

6. Cryan JF, Dinan TG. Mind-altering microorganisms: the impact of the gut microbiota on brain and behaviour. Nat Rev Neurosci. 2012;13(10):701-12.

7. Mohajeri MH, La Fata G, Steinert RE, Weber P. Relationship between the gut microbiome and brain function. Nutr Rev. 2018;76(7):481-96.

8. Ogbonnaya ES, Clarke G, Shanahan F, Dinan TG, Cryan JF, O'Leary OF. Adult hippocampal neurogenesis is regulated by the microbiome. Biol Psychiatry. 2015;78(4):e7-9.

9. Wang C, Zhu C, Shao L, Ye J, Shen Y, Ren Y. Role of bile acids in Dysbiosis and treatment of nonalcoholic fatty liver disease. Mediat Inflamm. 2019 . 2019:7659509.

10. Huang C, Wang J, Hu W, Wang C, Lu X, Tong L, Wu F, Zhang W. Identification of functional farnesoid $X$ receptors in brain neurons. FEBS Lett. 2016;590(18):3233-42.

11. Yanguas-Casas N, Barreda-Manso MA, Nieto-Sampedro M, Romero-Ramirez L. TUDCA: an agonist of the bile acid receptor GPBAR1/TGR5 with antiinflammatory effects in microglial cells. J Cell Physiol. 2017;232(8):2231-45.

12. Wu X, LV YG, Du YF, Chen F, Reed MN, Hu M, Suppiramaniam V, Tang SS, Hong $H$. Neuroprotective effects of INT-777 against Abeta1-42-induced cognitive impairment, neuroinflammation, apoptosis, and synaptic dysfunction in mice. Brain Behav Immun. 2018;73:533-45.

13. MahmoudianDehkordi S, Arnold M, Nho K, Ahmad S, Jia W, Xie G, Louie G, Kueider-Paisley A, Moseley MA, Thompson JW, et al. Altered bile acid profile associates with cognitive impairment in Alzheimer's disease-an emerging role for gut microbiome. Alzheimers Dement. 2019;15(1):76-92.

14. Jena PK, Sheng L, Nagar N, Wu C, Barile D, Mills DA, Wan YY. Synbiotics Bifidobacterium infantis and milk oligosaccharides are effective in reversing cancer-prone nonalcoholic steatohepatitis using western diet-fed FXR knockout mouse models. J Nutr Biochem. 2018;57:246-54. 
15. Mistry RH, Gu F, Schols HA, Verkade HJ, Tietge UJF. Effect of the prebiotic fiber inulin on cholesterol metabolism in wildtype mice. Sci Rep. 2018;8(1):13238.

16. Weitkunat K, Schumann S, Petzke KJ, Blaut M, Loh G, Klaus S. Effects of dietary inulin on bacterial growth, short-chain fatty acid production and hepatic lipid metabolism in gnotobiotic mice. J Nutr Biochem. 2015;26(9):929-37.

17. Smith AP, Sutherland D, Hewlett P. An investigation of the acute effects of Oligofructose-enriched inulin on subjective wellbeing, Mood and Cognitive Performance. Nutrients. 2015;7(11):8887-96.

18. Chunchai T, Keawtep P, Arinno A, Saiyasit N, Prus D, Apaijai N, Pratchayasakul W, Chattipakorn N, Chattipakorn SC. N-acetyl cysteine, inulin and the two as a combined therapy ameliorate cognitive decline in testosterone-deprived rats. Aging (Albany NY). 2019;11(11):3445-62.

19. Singh V, Yeoh BS, Chassaing B, Xiao X, Saha P, Aguilera Olvera R, Lapek JD Jr, Zhang L, Wang WB, Hao S, et al. Dysregulated Microbial Fermentation of Soluble Fiber Induces Cholestatic Liver Cancer. Cell. 2018;175(3):679-694.e622.

20. Wan Y-JY, Jena PK. Precision dietary supplementation based on personal gut microbiota. Nat Rev Gastroenterol Hepatol. 2019;16(4):204-6.

21. Sheng L, Jena PK, Liu HX, Kalanetra KM, Gonzalez FJ, French SW, Krishnan W, Mills DA, Wan YY. Gender differences in bile acids and microbiota in relationship with gender dissimilarity in Steatosis induced by diet and FXR inactivation. Sci Rep. 2017;7(1):1748

22. Bettaieb A, Jiang JX, Sasaki Y, Chao TI, Kiss Z, Chen X, Tian J, Katsuyama M, Yabe-Nishimura C, Xi Y, et al. Hepatocyte Nicotinamide Adenine Dinucleotide Phosphate Reduced Oxidase 4 Regulates Stress Signaling, Fibrosis, and Insulin Sensitivity During Development of Steatohepatitis in Mice. Gastroenterology. 2015;149(2):468-480.e410.

23. Kleiner $D E$, et al. Design and validation of a histological scoring system for nonalcoholic fatty liver disease. Hepatology. 2005;41(6):1313-21.

24. Jena PK, Sheng L, Di Lucente J, Jin LW, Maezawa I, Wan YY. Dysregulated bile acid synthesis and dysbiosis are implicated in Western diet-induced systemic inflammation, microglial activation, and reduced neuroplasticity. FASEB J. 2018;32(5):2866-77.

25. Williams JE, Carrothers JM, Lackey KA, Beatty NF, York MA, Brooker SL, Shafii B, Price WJ, Settles ML, McGuire MA, et al. Human Milk microbial community structure is relatively stable and related to variations in macronutrient and micronutrient intakes in healthy lactating women. J Nutr. 2017;147(9):1739-48.

26. Wang Q, Garrity GM, Tiedje JM, Cole JR. Naïve Bayesian Classifier for Rapid Assignment of rRNA Sequences into the New Bacterial Taxonomy. Appl Environ Microbiol. 2007;73(16):5261-7.

27. Sheng L, Jena PK, Liu H-X, Hu Y, Nagar N, Bronner DN, Settles ML, Bäumler AJ, Wan Y-JY. Obesity treatment by epigallocatechin-3-gallate-regulated bile acid signaling and its enriched Akkermansia muciniphila. FASEB J. 2018; 32(12):6371-84

28. Xia J, Wishart DS. Using MetaboAnalyst 3.0 for comprehensive metabolomics data analysis. Curr Protoc Bioinformatics. 2016;55:14.10.11-91.

29. Barupal DK, Fiehn O. Chemical similarity enrichment analysis (ChemRICH) as alternative to biochemical pathway mapping for metabolomic datasets. Sci Rep. 2017;7(1):14567.

30. Chong J, Wishart DS, Xia J. Using MetaboAnalyst 4.0 for Comprehensive and Integrative Metabolomics Data Analysis. Curr Protocols Bioinformatics. 2019; 68(1):e86.

31. Yu S, Wu X, Shi Z, Huynh M, Jena PK, Sheng L, Zhou Y, Han D, Wan YY, Hwang ST. Diet-induced obesity exacerbates imiquimod-mediated psoriasiform dermatitis in anti-PD-1 antibody-treated mice: implications for patients being treated with checkpoint inhibitors for cancer. J Dermatol Sci. 2020;97(3):194-200.

32. Shi Z, Wu X, Yu S, Huynh M, Jena PK, Nguyen M, Wan YY, Hwang ST. Shortterm exposure to a Western diet induces Psoriasiform dermatitis by promoting accumulation of IL-17A-producing $\gamma \delta$ T cells. J Invest Dermatol. 2020;140:1815

33. Jena PK, Sheng L, McNeil K, Chau TQ, Yu S, Kiuru M, Fung MA, Hwang ST, Wan YY. Long-term Western diet intake leads to dysregulated bile acid signaling and dermatitis with Th2 and Th17 pathway features in mice. J Dermatol Sci. 2019;95(1):13-20.

34. Cipollini V, Anrather J, Orzi F, ladecola C. Th17 and cognitive impairment: possible mechanisms of action. Front Neuroanat. 2019;13:95.

35. Zackular JP, Baxter NT, Iverson KD, Sadler WD, Petrosino JF, Chen GY, Schloss PD. The gut microbiome modulates colon tumorigenesis. MBio. 2013;4(6):e00692-13
36. Rizzatti G, Lopetuso LR, Gibiino G, Binda C, Gasbarrini A Proteobacteria: A Common Factor in Human Diseases. BioMed research international 2017: 2017:9351507

37. Hiippala K, Kainulainen V, Kalliomaki M, Arkkila P, Satokari R. Mucosa prevalence and interactions with the epithelium indicate commensalism of Sutterella spp. Front Microbiol. 2016;7:1706.

38. De Angelis M, Piccolo M, Vannini L, Siragusa S, De Giacomo A, Serrazzanetti DI, Cristofori F, Guerzoni ME, Gobbetti M, Francavilla R. Fecal microbiota and metabolome of children with autism and pervasive developmental disorder not otherwise specified. PLoS One. 2013;8(10):e76993.

39. Sheng L, Jena PK, Hu Y, Liu HX, Nagar N, Kalanetra KM, French SW, French SW, Mills DA, Wan YY. Hepatic inflammation caused by dysregulated bile acid synthesis is reversible by butyrate supplementation. J Pathol. 2017;243(4):431-41.

40. Duboc H, Tache $Y$, Hofmann AF. The bile acid TGR5 membrane receptor: from basic research to clinical application. Dig Liver Dis. 2014;46(4):302-12.

41. Keitel V, Gorg B, Bidmon HJ, Zemtsova I, Spomer L, Zilles K, Haussinger D. The bile acid receptor TGR5 (Gpbar-1) acts as a neurosteroid receptor in brain. Glia. 2010;58(15):1794-805

42. Gomes AL, Teijeiro A, Buren S, Tummala KS, Yilmaz M, Waisman A, Theurillat JP, Perna C, Djouder N. Metabolic inflammation-associated IL-17A causes non-alcoholic Steatohepatitis and hepatocellular carcinoma. Cancer Cell. 2016;30(1):161-75.

43. Meng F, Wang K, Aoyama T, Grivennikov SI, Paik Y, Scholten D, Cong M, Iwaisako K, Liu X, Zhang M, et al. Interleukin-17 signaling in inflammatory, Kupffer cells, and hepatic stellate cells exacerbates liver fibrosis in mice. Gastroenterology. 2012;143(3):765-776.e763.

44. Zhang J, Ke KF, Liu Z, Qiu YH, Peng YP. Th17 cell-mediated neuroinflammation is involved in neurodegeneration of abeta1-42-induced Alzheimer's disease model rats. PLoS One. 2013;8(10):e75786.

45. Qin H, Wang L, Feng T, Elson CO, Niyongere SA, Lee SJ, Reynolds SL, Weaver CT, Roarty K, Serra R, et al. TGF-beta promotes Th17 cell development through inhibition of SOCS3. J Immunol. 2009;183(1):97-105.

46. Hang S, Paik D, Yao L, Kim E, Trinath J, Lu J, Ha S, Nelson BN, Kelly SP, Wu L, et al. Bile acid metabolites control TH17 and Treg cell differentiation Nature. 2019;576:143-8

47. Shao CY, Mirra SS, Sait HB, Sacktor TC, Sigurdsson EM. Postsynaptic degeneration as revealed by PSD-95 reduction occurs after advanced Abeta and tau pathology in transgenic mouse models of Alzheimer's disease. Acta Neuropathol. 2011;122(3):285-92.

48. Bathina S, Das UN. Brain-derived neurotrophic factor and its clinical implications. Arch Med Sci. 2015;11(6):1164-78.

49. Meadows A, Lee JH, Wu CS, Wei Q, Pradhan G, Yafi M, Lu HC, Sun Y. Deletion of G-protein-coupled receptor 55 promotes obesity by reducing physical activity. Int J Obes. 2016;40(3):417-24.

50. Sharma S, Fulton S. Diet-induced obesity promotes depressive-like behaviour that is associated with neural adaptations in brain reward circuitry. Int J Obes. 2013;37(3):382-9.

51. Spencer MD, Hamp TJ, Reid RW, Fischer LM, Zeisel SH, Fodor AA Association between composition of the human gastrointestinal microbiome and development of fatty liver with choline deficiency. Gastroenterology. 2011;140(3):976-86.

52. Martinez I, Perdicaro DJ, Brown AW, Hammons S, Carden TJ, Carr TP, Eskridge KM, Walter J. Diet-induced alterations of host cholesterol metabolism are likely to affect the gut microbiota composition in hamsters. Appl Environ Microbiol. 2013;79(2):516-24.

53. Kain V, Pol WVD, Mariappan N, Ahmad A, Eipers P, Gibson DL, Gladine C, Vigor C, Durand T, Morrow C, et al. Obesogenic diet in aging mice disrupts gut microbe composition and alters neutrophil:lymphocyte ratio, leading to inflamed milieu in acute heart failure. FASEB J. 2019; 33(5):6456-69.

54. Tang W, Yao X, Xia F, Yang M, Chen Z, Zhou B, Liu Q. Modulation of the gut microbiota in rats by Hugan Qingzhi tablets during the treatment of highfat-diet-induced nonalcoholic fatty liver disease. Oxidative Med Cell Longev. 2018;2018:7261619.

55. Serino M, Luche E, Gres S, Baylac A, Bergé M, Cenac C, Waget A, Klopp P, lacovoni J, Klopp C, et al. Metabolic adaptation to a high-fat diet is associated with a change in the gut microbiota. Gut. 2012;61(4):543-53.

56. Harach T, Marungruang N, Duthilleul N, Cheatham V, Mc Coy KD, Frisoni G, Neher JJ, Fåk F, Jucker M, Lasser T, et al. Reduction of Abeta amyloid pathology in APPPS1 transgenic mice in the absence of gut microbiota. Sci Rep. 2017;7:41802 
57. Yadav $\mathrm{H}$, Jain $\mathrm{S}$, Sinha PR. Antidiabetic effect of probiotic dahi containing Lactobacillus acidophilus and Lactobacillus casei in high fructose fed rats. Nutrition. 2007;23(1):62-8.

58. Lew LC, Hor YY, Yusoff NAA, Choi SB, Yusoff MSB, Roslan NS, Ahmad A, Mohammad JAM, Abdullah M, Zakaria N, et al. Probiotic Lactobacillus plantarum P8 alleviated stress and anxiety while enhancing memory and cognition in stressed adults: a randomised, double-blind, placebo-controlled study. Clin Nutr. 2019;38(5):2053-64.

59. Ubeda C, Bucci V, Caballero S, Djukovic A, Toussaint NC, Equinda M, Lipuma L, Ling L, Gobourne A, No D, et al. Intestinal microbiota containing Barnesiella species cures vancomycin-resistant Enterococcus faecium colonization. Infect Immun. 2013;81(3):965-73.

60. Yang F, Huang X, Yi T, Yen Y, Moore DD, Huang W. Spontaneous development of liver tumors in the absence of the bile acid receptor farnesoid X receptor. Cancer Res. 2007:67(3):863-7.

61. Alawad AS, Levy C. FXR agonists: from bench to bedside, a guide for clinicians. Dig Dis Sci. 2016;61(12):3395-404.

62. McMillin M, Frampton G, Tobin R, Dusio G, Smith J, Shin H, Newell-Rogers K, Grant S, DeMorrow S. TGR5 signaling reduces neuroinflammation during hepatic encephalopathy. J Neurochem. 2015;135(3):565-76.

63. Hubbard TD, Liu Q, Murray IA, Dong F, Miller C 3rd, Smith PB, Gowda K, Lin $J \mathrm{M}$, Amin $\mathrm{S}$, Patterson $\mathrm{AD}$, et al. Microbiota metabolism promotes synthesis of the human ah receptor agonist 2,8-Dihydroxyquinoline. J Proteome Res. 2019;18(4):1715-24.

64. Kowalik MA, Columbano A, Perra A. Emerging role of the pentose phosphate pathway in hepatocellular carcinoma. Front Oncol. 2017;7:87.

65. Wu G, Bazer FW, Burghardt RC, Johnson GA, Kim SW, Knabe DA, Li P, Li X, McKnight JR, Satterfield MC, et al. Proline and hydroxyproline metabolism: implications for animal and human nutrition. Amino Acids. 2011;40(4):1053-63.

66. Ikeda I, Sugano M. Some aspects of mechanism of inhibition of cholesterol absorption by beta-sitosterol. Biochim Biophys Acta. 1983;732(3):651-8.

67. Schommer J, Marwarha G, Nagamoto-Combs K, Ghribi O. Palmitic acidenriched diet increases a-Synuclein and tyrosine hydroxylase expression levels in the mouse brain. Front Neurosci. 2018;12:552.

\section{Publisher's Note}

Springer Nature remains neutral with regard to jurisdictional claims in published maps and institutional affiliations.

Ready to submit your research? Choose BMC and benefit from:

- fast, convenient online submission

- thorough peer review by experienced researchers in your field

- rapid publication on acceptance

- support for research data, including large and complex data types

- gold Open Access which fosters wider collaboration and increased citations

- maximum visibility for your research: over $100 \mathrm{M}$ website views per year

At BMC, research is always in progress.

Learn more biomedcentral.com/submissions 